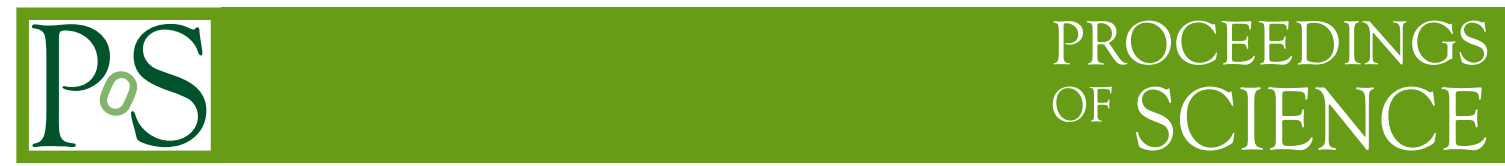

\title{
Spectroscopy at LHCb - exotic states
}

\author{
Liming Zhang ${ }^{* \dagger}$ \\ Center for High Energy Physics, Tsinghua University, Beijing, China \\ E-mail: liming.zhang@cern.ch
}

The most recent LHCb results related to spectroscopy of exotic states are reported, including an update of the $J / \psi p$ resonances where a new lighter narrow state has been discovered, evidence of charged $Z_{c}^{-} \rightarrow \eta_{c} \pi^{-}$state, and observation of decays of $B_{(s)}^{0} \rightarrow J / \psi p \bar{p}$, which is useful for exotic state searches.

Beauty 2019

29 Sep. - 4 Oct. 2019

Ljubljana

*Speaker.

${ }^{\dagger}$ on behalf of the LHCb Collaboration 


\section{Introduction}

Hadron spectroscopy is the study of the meson and baryon spectra and the search for exotics. Like atomic spectra where precise details led to the development of Quantum Electrodynamics (QED), hadron spectra beautifully encode the complex properties of Quantum Chromodynamics (QCD). Meson and baryon spectra are ideal place to the study quark-quark interaction and colour degrees of freedom. Exotic states, with quark configurations other than three quarks or quarkantiquark, can reveal new or hidden aspects of the dynamics of the strong interactions, which are described by QCD.

While QCD is well tested at high energies, where the strong coupling constant $\alpha_{s}$ is sufficiently small for perturbation theory to apply, it becomes a strongly coupled theory in the low energy regime where many aspects await a better understanding. Because the interaction in quark-formed hadrons happens at this regime, extensive and precise spectroscopy, particularly of exotic states, combined with a thorough theoretical analysis of the data, will add substantially to our knowledge.

Here, I discuss the most recent studies on exotic states performed by the LHCb collaboration.

\section{Recent pentaquark results from $\Lambda_{b}^{0} \rightarrow J / \psi p K^{-}$decays}

Hadrons made of four quarks and one antiquark, called pentaquarks, are allowed by QCD. In 2015 the LHCb experiment reported an observation of $J / \psi p$ resonant structures [1, 2], consistent with charmonium pentaquark with a minimal quark content of $\bar{c} c u u d$, produced in decays of $\Lambda_{b}^{0} \rightarrow J / \psi p K^{-}$(Charge conjugation is implied throughout the proceedings). The initial observation indicated a broad structure $P_{c}(4380)^{+}$and a narrow peak $P_{c}(4450)^{+}$. It is a major turning point in exotic baryon spectroscopy.

In 2019, a refined picture was presented. The peak structure at $4450 \mathrm{MeV}$ was confirmed but found to be a composition of two narrower peaks, $P_{c}(4440)^{+}$and $P_{c}(4457)^{+}$, with almost identical masses. In addition, a new narrow peak $P_{c}(4312)^{+}$at a lower mass was also uncovered [3]. As the analysis did not incorporate decay angular distributions, no confirmation of the $P_{c}(4380)^{+}$was possible.

Compared to the 2015 results, the 2019 ones [3] include the additional run 2 data, corresponding to an integrated luminosity of $6 \mathrm{fb}^{-1}$ in $13 \mathrm{TeV} p p$ collisions. The selected $\Lambda_{b}^{0}$ signal yield is 246000 , which is almost an order of magnitude higher than that obtained in the earlier result [1]. The large yield is achieved thanks to improvements in the data selection, which provide a factor of 2 increase and keep signal purity at a similar level (93.6\%), and the additional run 2 data, which gives a factor of 5 increase. When applying the same amplitude model to the new data sample, consistent parameters for the $P_{c}(4380)^{+}$and $P_{c}(4450)^{+}$states with the 2015 results are obtained. This check provides an important confirmation for the previous LHCb's pentaquark study, since an almost statistically independent sample is used.

However this should be just taken as a cross-check, because the new high statistic data reveals more $J / \psi p$ structures, which were too small to be significant before. The invariant mass spectra of $p K^{-}$and $J / \psi p$ of selected $\Lambda_{b}^{0}$ candidates are shown in Fig. 1 , with a bin size of $2 \mathrm{MeV}$, which is comparable to the mass resolution. A narrow peak is observed near $4312 \mathrm{MeV}$. The structure at $4450 \mathrm{MeV}$ is now resolved into two narrow peaks at 4440 and $4457 \mathrm{MeV}$. 

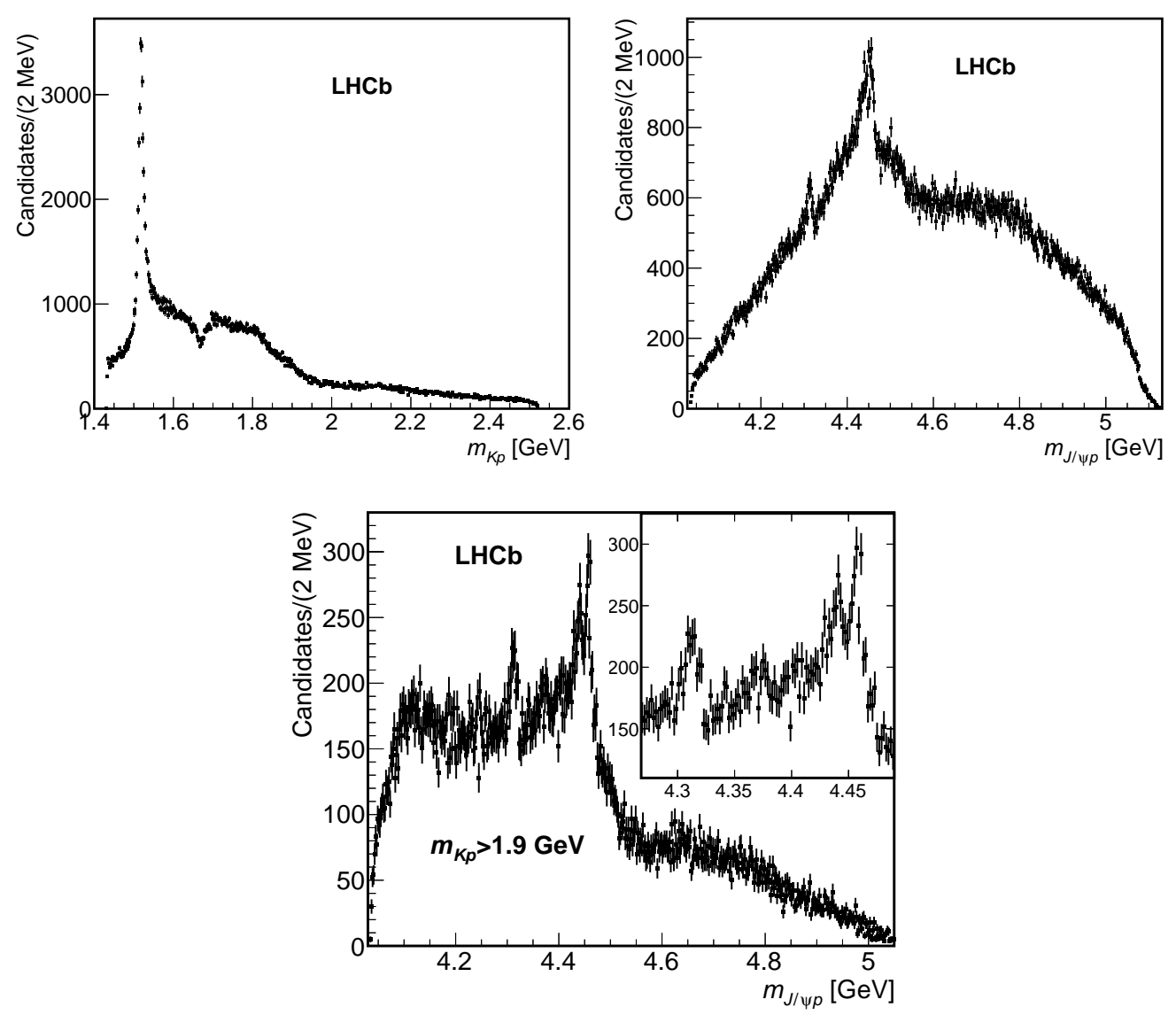

Figure 1: Distribution of (top left) $m_{k p}$ and (top right) $m_{J / \psi p}$ for the selected $\Lambda_{b}^{0} \rightarrow J / \psi p K^{-}$candidates from Ref. [3]. (bottom) Distribution of $m_{J / \psi p}$ with $m_{K p}>1.9 \mathrm{GeV}$ requirement to reduce the $\Lambda^{*}$ background. A narrow peak is observed near $4312 \mathrm{MeV}$. The structure at $4450 \mathrm{MeV}$ is now resolved into two narrow peaks at 4440 and $4457 \mathrm{MeV}$.

To study the narrow structures, LHCb performed an analysis that fits the one-dimensional $m_{J / \psi p}$ distribution. Such analysis is insensitive to any broad pentaquark structure, like the $P_{c}(4380)^{+}$ state, and is also insensitive to the spin-parities of the narrow pentaquark structures. The effect of the interference between narrow $P_{c}^{+}$with the other components (either another $P_{c}^{+}$states or $\Lambda^{*}$ resonances) is found to be small, therefore the analysis can robustly determine the masses and widths for the narrow $P_{c}^{+}$states. In the nominal fit, the invariant mass distribution of $m_{J / \psi p}$ is fitted by three Breit-Wigner functions smeared by the mass resolution $(2-3 \mathrm{MeV})$, plus a high-order polynomial to account for any other contributions, i.e. the $\Lambda^{*}$ reflections, no- $\Lambda_{b}^{0}$ sideband background and possible broad $P_{c}^{+}$component. To estimate systematic uncertainties, three $m_{J / \psi p}$ distributions with different selection or weighting procedure are fitted: total candidates under the $\Lambda_{b}^{0}$ peak, $m_{K p}>1.9 \mathrm{GeV}$ requirement to reduce the $\Lambda^{*}$ background, and total candidates with $\cos \theta_{P_{c}}$ dependent weighting that optimally suppresses the $\Lambda^{*}$ background, where $\theta_{P_{c}}$ is helicity angle of $P_{c}^{+} \rightarrow J / \psi p$ decay. Figure 2 shows the fit with the $\cos \theta_{P_{c}}$ dependent weights, which gives the nominal fit values. The results for the masses, widths and relative production rates $(\mathscr{R})$ are given in 
Table 1: Summary of $P_{c}^{+}$properties [3].

\begin{tabular}{c|c|rc|c} 
State & $M[\mathrm{MeV}]$ & \multicolumn{1}{c}{$\Gamma[\mathrm{MeV}]$} & $(95 \% \mathrm{CL})$ & $\mathscr{R}[\%]$ \\
\hline$P_{c}(4312)^{+}$ & $4311.9 \pm 0.7_{-0.6}^{+6.8}$ & $9.8 \pm 2.7_{-4.5}^{+3.7}$ & $(<27)$ & $0.30 \pm 0.07_{-0.09}^{+0.34}$ \\
$P_{c}(4440)^{+}$ & $4440.3 \pm 1.3_{-4.7}^{+4.1}$ & $20.6 \pm 4.9_{-10.1}^{+8.7}$ & $(<49)$ & $1.11 \pm 0.33_{-0.10}^{+0.22}$ \\
$P_{c}(4457)^{+}$ & $4457.3 \pm 0.6_{-1.7}^{+4.1}$ & $6.4 \pm 2.0_{-1.9}^{+5.7}$ & $(<20)$ & $0.53 \pm 0.16_{-0.13}^{+0.15}$ \\
\hline
\end{tabular}

Table 1. Throughout the proceedings, whenever uncertainties are shown, the first is the statistical and the second is the systematic. The widths of these states are quite narrow. The significance of the $P_{c}(4312)^{+}$state is more than $7.3 \sigma$. The significance of the two-peak versus one-peak hypothesis for the $4450 \mathrm{MeV}$ structure is over $5.4 \sigma$.

The observed three $P_{c}^{+}$states are just several to tens $\mathrm{MeV}$ below the $\Sigma_{c}^{+} \bar{D}^{(*) 0}$ threshold. The mass values coincidence with two relative thresholds, as well as the very narrow widths of these states favour the loosely-bound ("molecular") pentaquark model. However the other explanations are not firmly ruled out. To give a firm conclusion, spin-parity measurements are necessary, which require a detailed amplitude analysis as done in 2015 [1].

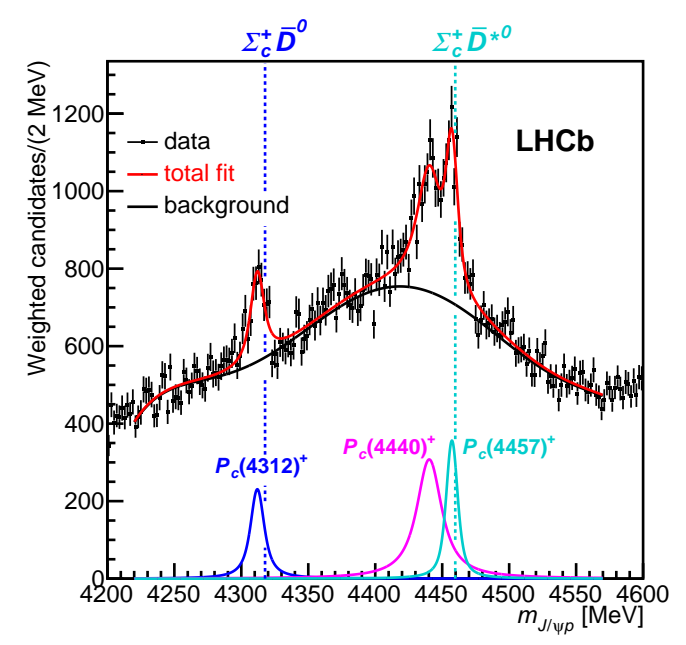

Figure 2: Fit to weighted $m_{J / \psi p}$ distribution of the selected $\Lambda_{b}^{0} \rightarrow J / \psi p K^{-}$candidates from Ref. [3]. Three narrow peak structures are modelled by Breit-Wigner function smeared by resolution, the background is described by high order polynomial function. The two vertical dashed lines indict $\Sigma_{c}^{+} \bar{D}^{0}$ and $\Sigma_{c}^{+} \bar{D}^{* 0}$ mass thresholds.

\section{Evidence of a $Z_{c}^{-} \rightarrow \eta_{c} \pi^{-}$resonance at $\mathrm{LHCb}$}

Many charged charmonium-like states are confirmed as or consistent with being $J^{P}=1^{+}$, including $Z_{c}(3900)^{+}, Z_{c}(4020)^{+}, Z(4200)^{+}$and $Z(4430)^{+}$. While the $\eta_{c} \pi^{ \pm}$final states can access $J^{P}=0^{+}, 1^{-}$and $2^{+}$, and therefore are sensitive to new states.

The LHCb experiment performed a Dalitz-plot analysis to $B^{0} \rightarrow \eta_{c}(1 S) K^{+} \pi^{-} ; \eta_{c}(1 S) \rightarrow$ $p \bar{p}$ decays with their run-1 and 2015-2016 data, corresponding to an integrated luminosity of 
$4.7 \mathrm{fb}^{-1}$ [4]. A two-dimensional fit to distributions of $p \bar{p} K^{+} \pi^{-}$and $p \bar{p}$ of selected candidates yields $1870 \pm 74$ signal decays. The decay is expected to be dominated by intermediate resonances of various kaon excitations, in which several $K^{* 0}$ resonances are found to be significant. The data show a bump in the $\eta_{c} \pi^{-}$invariant mass distribution at about $4.1 \mathrm{GeV}$ that cannot be described by the fit. Then a $Z_{c}^{-}$state with $J^{P}=0^{+}, 1^{-}$and $2^{+}$is added in turn to the amplitude fit. Twice the $\log$ likelihood $(2 \ln \mathscr{L})$ is improved by $22.8,41.4$ and 7.0 , respectively. The largest value of the improvement corresponds to a $4.8 \sigma$ statistical significance. Figure 3 shows the best fit. Significances of the state and spin-parity assignment are reduced when considering systematic uncertainties. When varying systematic conditions, the smallest significance for the $1^{-}$assignment is $3.4 \sigma$. The discrimination between the $J^{P}=0^{+}$and $1^{-}$assignments is reduced to only $1.2 \sigma$, when varying the model for $S$-wave $K^{*}$ component. The charged charmonium-like state is found to have $m_{0}=$ $4096 \pm 20_{-22}^{+18} \mathrm{MeV}$ and width $152 \pm 58_{-35}^{+60} \mathrm{MeV}$, respectively, thus called $Z_{c}(4100)^{-}$. The product branching fraction is also measured to be $\mathscr{B}\left(B^{0} \rightarrow Z_{c}(4100)^{-} K^{+}, Z_{c}(4100)^{-} \rightarrow \eta_{c}(1 S) \pi^{-}\right)=$ $\left(1.89 \pm 0.64_{-0.67}^{+0.73}\right) \times 10^{-5}[4]$.

Interestingly, the result of $Z_{c}(4100)^{-}$state is consistent with $Z(4050)^{-} J^{P}=0^{+}$state, $m_{0}=$ $4051_{-40}^{+24} \mathrm{MeV}$ and width $82_{-28}^{+50} \mathrm{MeV}$, observed by the Belle experiment in $\chi_{c 1} \pi^{-}$final state also from $B^{0}$ decays [5]. Are the two states the same one? More studies are needed.
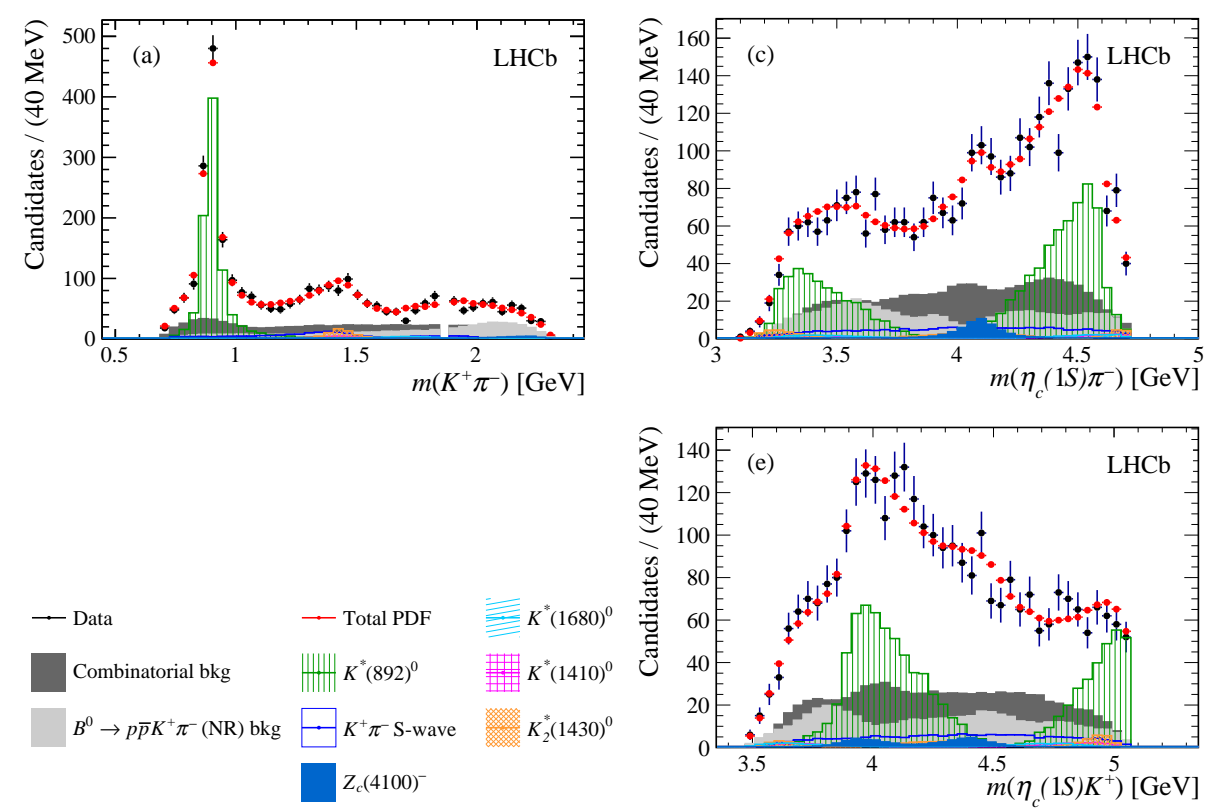

Figure 3: Fit projections of invariant masses for (top left) $K^{+} \pi^{-}$(top right) $\eta_{c}(1 S) \pi^{-}$and (bottom right) $\eta_{c}(1 S) K^{+}$from the $B^{0} \rightarrow \eta_{c}(1 S) K^{+} \pi^{-} ; \eta_{c}(1 S) \rightarrow p \bar{p}$ candidates [4].

\section{Observation of $B_{(s)}^{0} \rightarrow J / \psi p \bar{p}$ decays}

The decay $B_{(s)}^{0} \rightarrow J / \psi p \bar{p}$ is sensitive to exotic states in $J / \psi p$ system and Glueballs in $p \bar{p}$ system. The naive theoretical prediction for the branching fraction of $B_{s}^{0} \rightarrow J / \psi p \bar{p}$ decay is $\approx 10^{-9}[6]$. 
With the LHCb data sample corresponding to $5.2 \mathrm{fb}^{-1}$ integrated luminosity, both decays are observed [7]. The invariant mass distribution of $J / \psi p \bar{p}$ is shown in Fig. 4. A fit to the distribution returns $256 \pm 22 B^{0}$ and $609 \pm 31 B_{s}^{0}$ signal decays. The resulting branching fractions are measured to be

$$
\begin{aligned}
& \mathscr{B}\left(B^{0} \rightarrow J / \psi p \bar{p}\right)=(4.51 \pm 0.40 \pm 0.44) \times 10^{-7}, \\
& \mathscr{B}\left(B_{s}^{0} \rightarrow J / \psi p \bar{p}\right)=(3.58 \pm 0.19 \pm 0.39) \times 10^{-6} .
\end{aligned}
$$

The large signal yield of $B_{s}^{0}$ decays allow a full amplitude analysis, which is in progress.

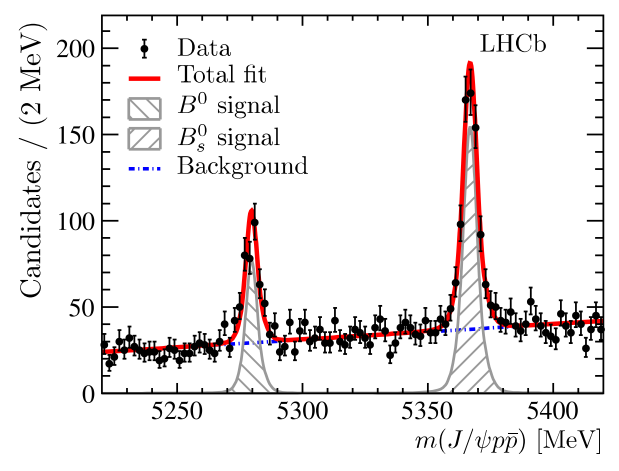

Figure 4: Fit to the invariant mass distribution of $J / \psi p \bar{p}$ [7].

\section{Summary}

In summary, many studies of exotic states have been performed by the LHCb experiments at high energy $p p$ collisions. With almost ten time larger $\Lambda_{b}^{0} \rightarrow J / \psi p K^{-}$decays than that observed in 2015, three narrow pentaquark states are observed. The fact that their masses are just several to tens $\mathrm{MeV}$ below $\Sigma_{c}^{+} \bar{D}^{(*) 0}$ hadron thresholds, suggests the thresholds must play an important role in the dynamics of these states. An evident $\eta_{c} \pi^{-}$exotic structure is obtained in $B^{0} \rightarrow \eta_{c} K^{+} \pi^{-}$ decays. In addition, branching fraction of $B_{s}^{0} \rightarrow J / \psi p \bar{p}$ is measured to be several magnitude higher than the naive prediction, providing a good opportunity to study the underlying contributions to this decay.

\section{References}

[1] LHCb collaboration, R. Aaij et al., Phys. Rev. Lett. 115 (2015) 072001, arXiv:1507. 03414.

[2] LHCb collaboration, R. Aaij et al., Phys. Rev. D95 (2017) 012002, arXiv:1606. 07898.

[3] LHCb collaboration, R. Aaij et al., Phys. Rev. Lett. 122 (2019) 222001, arXiv: 1904.03947.

[4] LHCb collaboration, R. Aaij et al., Eur. Phys. J. C78 (2018) 1019, arXiv:1809. 07416.

[5] Belle collaboration, R. Mizuk et al., Phys. Rev. D78 (2008) 072004, arXiv: 0806.4098.

[6] Y. K. Hsiao and C. Q. Geng, Eur. Phys. J. C75 (2015), no. 3 101, arXiv: 1412.4900.

[7] LHCb collaboration, R. Aaij et al., Phys. Rev. Lett. 122 (2019) 191804, arXiv: 1902.05588. 